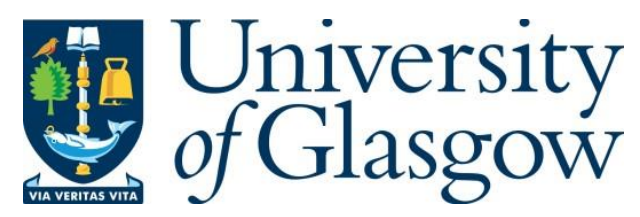

Casey-Bryars, M. et al. (2018) Waves of endemic foot-and-mouth disease in eastern Africa suggest feasibility of proactive vaccination approaches. Nature Ecology and Evolution, 2(9), pp. 1449-1457. (doi:10.1038/s41559-018-0636-x)

There may be differences between this version and the published version. You are advised to consult the publisher's version if you wish to cite from it.

http://eprints.gla.ac.uk/167483/

Deposited on: 27 September 2018

Enlighten - Research publications by members of the University of Glasgow http://eprints.gla.ac.uk 


\title{
Waves of endemic foot-and-mouth disease in eastern Africa suggest feasibility of proactive vaccination approaches
}

\author{
Miriam Casey-Bryars ${ }^{1,2,3,+}$, Richard Reeve ${ }^{1,+}$, Umesh Bastola ${ }^{4}$, Nick J. Knowles ${ }^{3}$, Harriet \\ Auty $^{5}$, Katarzyna Bachanek-Bankowska ${ }^{3}$, Veronica L. Fowler ${ }^{3}$, Robert Fyumagwa $^{6}$, \\ Rudovick Kazwala $^{7}$, Tito Kibona ${ }^{7,8}$, Alasdair King ${ }^{9}$, Donald P. King ${ }^{3}$, Felix Lankester ${ }^{1, \#, ~ A n n a ~}$ \\ B. Ludi ${ }^{3}$, Ahmed Lugelo ${ }^{7}$, Francois F. Maree $^{10}$, Deogratius Mshanga ${ }^{11}$, Gloria Ndhlovu ${ }^{7}$, \\ Krupali Parekh ${ }^{3}$, David J. Paton ${ }^{3}$, Brian Perry ${ }^{12,13}$, Jemma Wadsworth ${ }^{3}$, Satya Parida ${ }^{3}$, \\ Daniel T. Haydon ${ }^{1}$, Thomas L. Marsh ${ }^{4}$, Sarah Cleaveland ${ }^{1}$, and Tiziana Lembo ${ }^{1, *}$
}

${ }^{+}$These authors contributed equally to this work

*Corresponding author: tiziana.lembo@glasgow.ac.uk

${ }^{1}$ Boyd Orr Centre for Population and Ecosystem Health, Institute of Biodiversity, Animal Health and Comparative Medicine, College of Medical, Veterinary and Life Sciences, University of Glasgow, UK. ${ }^{2}$ Department of Agriculture, Food and the Marine, Ireland. ${ }^{3}$ The Pirbright Institute, Ash Road, Pirbright, Surrey, GU24 0NF, UK. ${ }^{4}$ School of Economic Sciences and Paul G. Allen School for Global Animal Health, Washington State University, USA. ${ }^{5}$ Scotland's Rural College, Beechwood Campus, An Lòchran, Inverness, IV2 5NA, UK. ${ }^{6}$ Tanzania Wildlife Research Institute, Arusha, Tanzania. ${ }^{7}$ Sokoine University of Agriculture, Morogoro, Tanzania. ${ }^{8}$ Nelson Mandela African Institution of Science and Technology, Arusha, Tanzania. ${ }^{9}$ Merck Animal Health (known as MSD Animal Health outside USA and Canada), Madison, New Jersey, USA. ${ }^{10}$ Agricultural Research Council, Onderstepoort Veterinary Institute, South Africa. ${ }^{11}$ Tanzania Veterinary Laboratory Agency, Ministry of Livestock and Fisheries, Arusha, Tanzania. ${ }^{12}$ College of Medicine and Veterinary Medicine, University of Edinburgh, UK. ${ }^{13}$ Nuffield Department of Clinical Medicine, University of Oxford, UK. "Currently at Paul G. Allen School for Global Animal Health, Washington State University, USA.

\section{Abstract}

Livestock production in Africa is key to national economies, food security and rural livelihoods, and $>85 \%$ of livestock keepers live in extreme poverty. With poverty elimination central to the Sustainable Development Goals, livestock keepers are therefore critically important. Foot-and-mouth disease (FMD) is a highly contagious livestock disease widespread in Africa that contributes to this poverty. Despite its $\$ 2.3 \mathrm{~B}$ impact, control of the disease is not prioritised: standard vaccination regimes are too costly, its impact on the poorest is underestimated, and its epidemiology is too weakly understood. Our integrated analysis in Tanzania shows that the disease is of high concern, reduces human health budgets and has major impacts on milk production and draft power for crop production. Critically, FMD outbreaks in cattle are driven by livestock-related factors with a pattern of changing serotype dominance over time. Contrary to findings in southern Africa, we find no evidence of frequent infection from wildlife, with outbreaks in cattle sweeping slowly across the region through a sequence of dominant serotypes. This regularity suggests that timely identification of the epidemic serotype could allow proactive vaccination ahead of the wave of infection, mitigating impacts, and our preliminary matching work has identified potential vaccine candidates. This strategy is more realistic than wildlife-livestock separation or conventional FMD vaccination approaches. Overall, we provide strong evidence for the feasibility of coordinated FMD control as part of livestock development policies in eastern Africa, and our integrated socio-economic, epidemiological, laboratory and modelling approach provides a framework for the study of other disease systems. 


\section{Introduction}

FMD in Africa involves five (O, A, and Southern African Territories [SAT] 1, 2 and 3) of the seven serotypes ${ }^{1}$ and multiple susceptible host species ${ }^{2}$. An incomplete understanding of its complex epidemiology constrains our ability to implement control suitable for the continent and contribute to the global strategy of the Food and Agriculture Organization (FAO) and World Organisation for Animal Health (OIE) for FMD (the Progressive Control Pathway for FMD, PCP-FMD ${ }^{3,4}$ ). Livestock are essential for food security, livelihoods, cultural identity and social status of small-scale farmers in Africa ${ }^{5,6}$, but endemic FMD circulation stifles economic growth and productivity, affecting food and economic security of the poorest families ${ }^{5,7}$. Economic losses from FMD include direct production losses alone of US $\$ 2.3$ billion per year ${ }^{8}$, over $0.1 \%$ of sub-Saharan Africa's entire Gross Domestic Product ${ }^{9}$ (where the disease is predominantly found), and indirect losses through restrictions on Africa's economic growth by impeding domestic and international trade ${ }^{10}$ (where indirect losses to an economy from FMD can starkly overshadow production losses). Although these impacts have been described at an aggregate level, control policies targeting those most affected require an understanding of household-level impacts, incentives, and heterogeneities across production systems and regions.

Policy on the continent has been largely driven by the southern African experience, where farming is more industrialised, making control by mass, regular vaccination affordable. As a result, FMD is endemic only in wildlife, and the African buffalo (Syncerus caffer) is an important source of foot-and-mouth disease virus (FMDV) for livestock ${ }^{11}$. In this setting, control now relies on separation of livestock and wildlife with zonal vaccination in neighbouring areas ${ }^{12}$ to prevent re-emergence in livestock. In eastern Africa, a separation-based approach is less viable because ecosystem integrity, vital for national economies, depends substantially on animal movements. Moreover, the importance of wildlife in the epidemiology of FMD in eastern Africa is less well documented. In other continents, where wildlife has not been implicated in the epidemiology of the disease, livestock vaccination has been successful in controlling FMD ${ }^{4,13}$, although conventional mass and ring vaccination approaches may be less effective in Africa because of a lack of resources and inadequate controls on livestock movements. The temporal and spatial dynamics of different serotypes and their lineages must also be investigated, otherwise antigenic matching cannot be used to determine whether appropriate vaccines are available as an intervention option ${ }^{14}$.

In this article, we investigate FMD in eastern Africa, with a particular focus on Tanzania because it has the highest density of African buffalo in Africa ${ }^{15}$, which live in close proximity to highdensity livestock populations (Fig. 1). Northern Tanzania is also representative of traditional livestock production systems, which are the most heavily impacted. We therefore target Tanzanian livestockowning communities and sympatric buffalo populations to quantify the impacts of FMD on livestock production and household decisions, and explore the drivers of FMD circulation in livestock-wildlife interface areas. We investigate outbreaks in the communities, and characterise the viruses isolated, studying serotype-specific circulation patterns, both here and in the rest of eastern Africa where data are available, to identify suitable vaccines and make best use of vaccine-based control options ${ }^{16}$. Finally, we explore the feasibility, and policy- and community-level acceptability of livestock vaccination. 


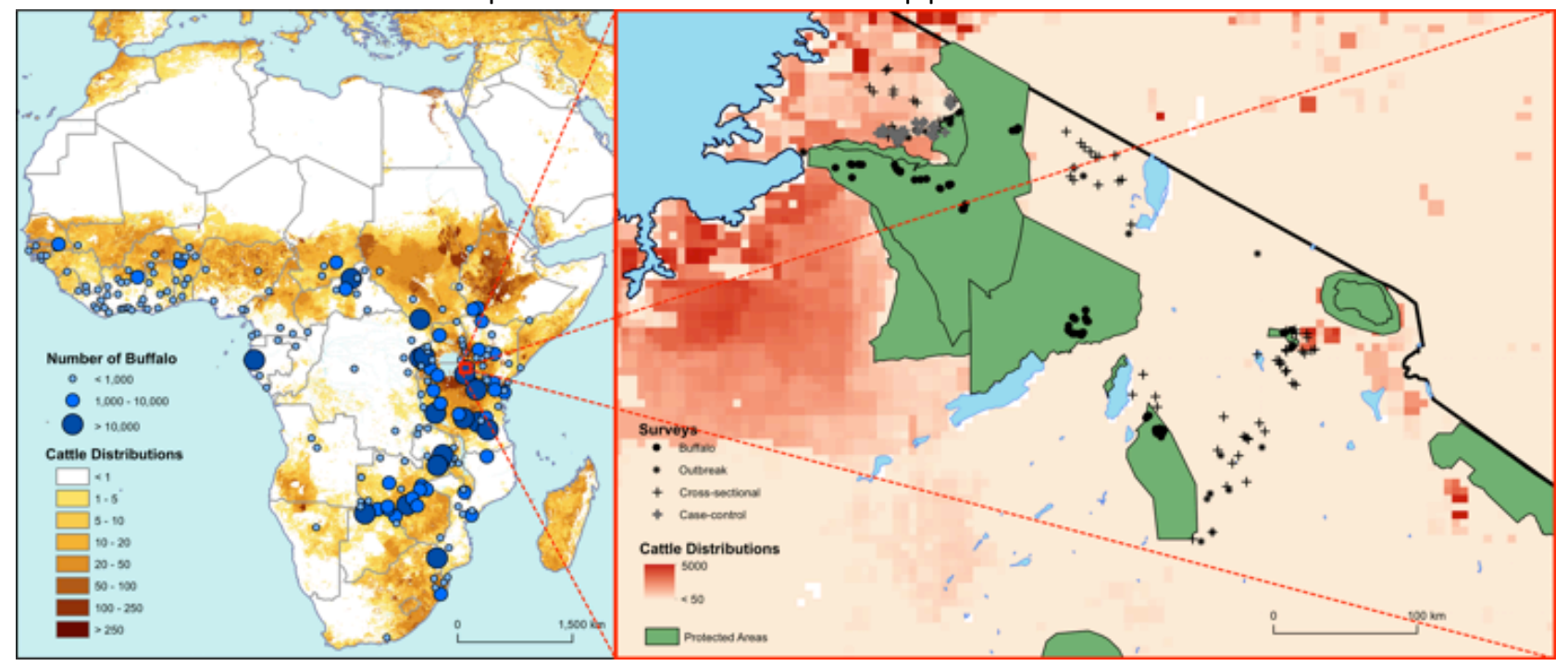

Figure 1: Study area. A map of the study area in northern Tanzania (right) annotated with locations of surveys (symbols), protected areas (green) including national parks (NPs), districts (Ds - Arusha and Arusha Urban are grouped together) and cattle density ${ }^{17}$ (red shading), located within a map of Africa (left) annotated with buffalo and cattle densities. The plot on the left shows cattle density ${ }^{18}$ and buffalo numbers ${ }^{15}$ in Africa.

\section{Results}

Household-level impacts To quantify household-level FMD impacts, we conducted cross-sectional, longitudinal and outbreak questionnaire studies as well as pan-serotypic non-structural protein (NSP) antibody serological surveys across different livestock management systems in nine districts across three regions of northern Tanzania (Fig. 1).

These systems included pastoral, agro-pastoral and rural smallholders, where (among 100 respondents) sales of livestock, crops and milk were the main sources of income (Supplementary Table 1). Microeconometric models were applied to determine FMD impacts on household production, income, and expenditures. Frequent FMD outbreaks were reported in most pastoralist and agro-pastoralist households (up to three/year), but less frequently amongst rural smallholders (Supplementary Table 2). Survival analyses of outbreaks in 37 longitudinally-tracked herds indicated a median time between outbreaks of 489 days (interquartile range 351-859 days, Fig. 2a), with four herds experiencing four outbreaks in less than three years. Similarly, seropositivity in livestock was higher in pastoralist and agro-pastoralist than rural smallholder systems (Supplementary Table 2). Consistent with other studies ${ }^{19,20}$, FMD was the disease of greatest concern to agro-pastoralists, ranked second by pastoralists, and was also of concern to rural smallholders (Fig. 2b). Although overall mortality levels were low (Supplementary Table 2), morbidity impacts were wide-ranging, with lactating cows being especially affected followed by other adult cattle (Fig. 2c). This is important since children in this area are vulnerable to undernutrition and stunting, and particularly reliant on milk as a protein source ${ }^{21}$. Although young stock might be expected to be particularly susceptible, the fact that they were not reported as being the most affected may be because of less obvious clinical signs, and for older cattle, concern has been expressed about potential fertility issues that may lead to further production losses ${ }^{22}$. FMD was associated with considerably lower herd milk yield (mean percentage decrease $67 \%$, Paired t-test: $\mathrm{p}<10^{-6}$ ), with $90 \%$ of respondents reporting reduced cow milk production during outbreaks (Fig. 2d). Similarly, decreased goat milk was reported by $65 \%$ of respondents. The majority (63\%) therefore stopped selling milk and $26 \%$ of households stopped consuming it (Supplementary Table 2). As a means of self-insurance against milk loss, households with FMD retained 10\% more lactating cows (Supplementary Table 3a). A loss of traction capacity 


\section{Waves of endemic foot-and-mouth disease in eastern Africa suggest feasibility of}

proactive vaccination approaches

affected $73 \%$ of all households, with $65 \%$ reporting that this negatively impacted crop production. FMD outbreaks decreased cash generation from livestock sales in our sample by an average of $27 \%$ (US $\$ 234 /$ household), reducing expenditure on human health by $25 \%$ (US $\$ 3.13 /$ household member, Supplementary Table 4).

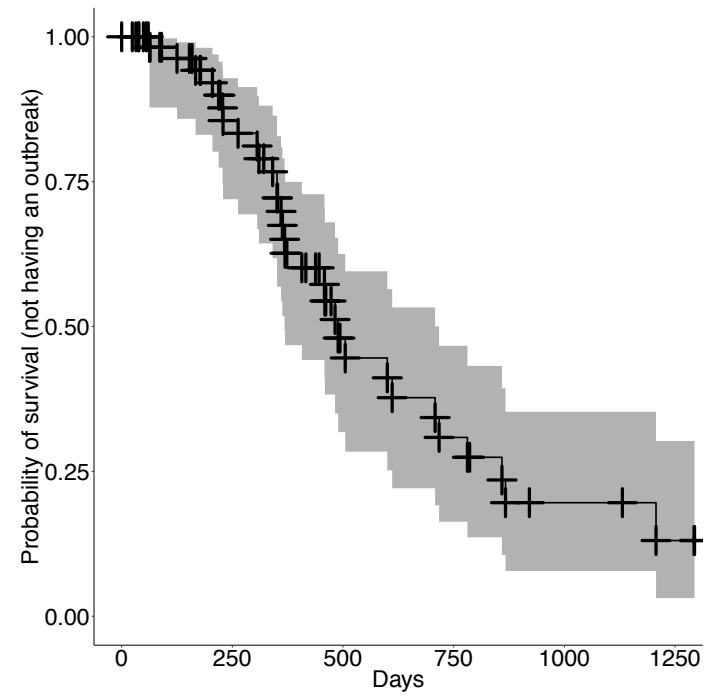

C

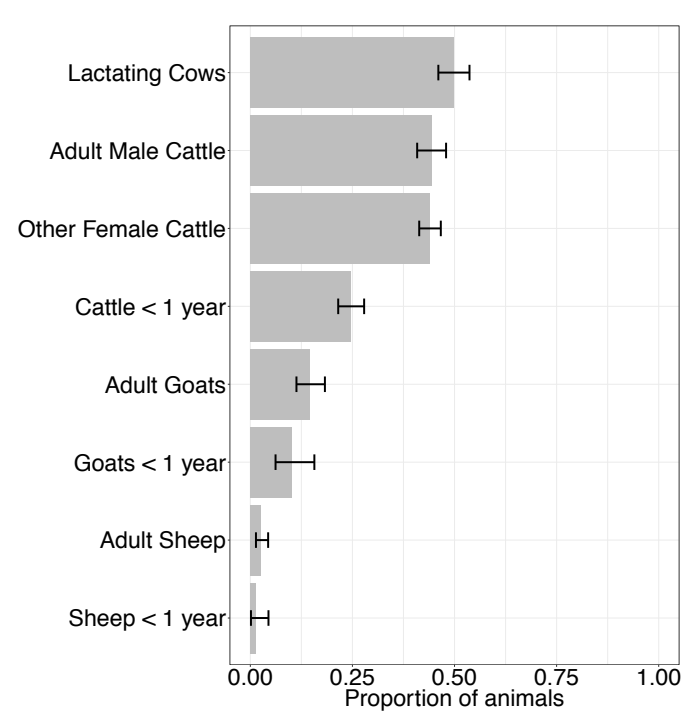

b

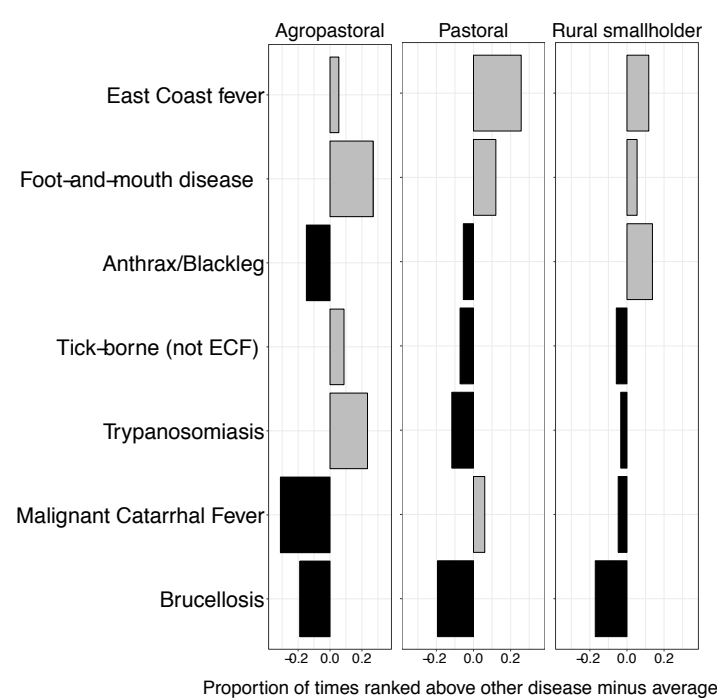

d

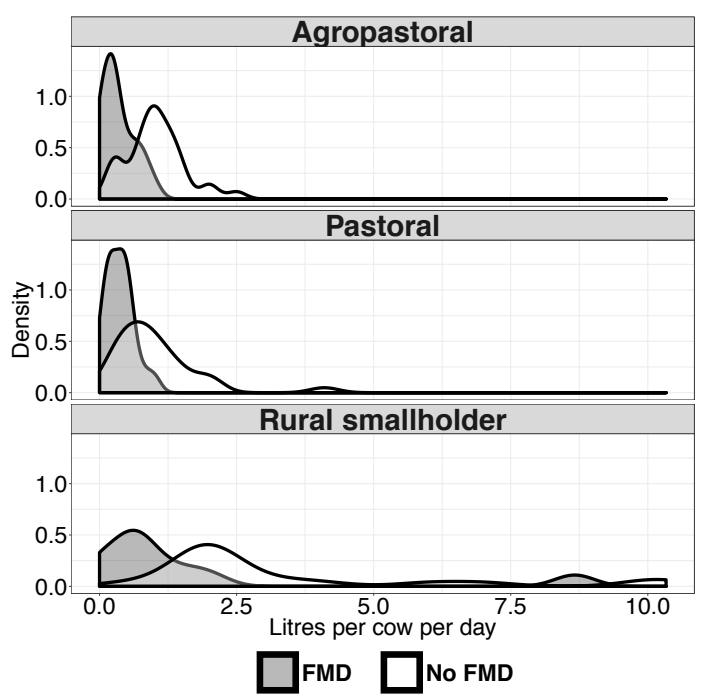

Figure 2: Household-level impacts of foot-and-mouth disease. a. Kaplan Meyer curve showing estimation of the time between FMD outbreaks in longitudinally tracked herds. The y-axis shows the probability of not having an outbreak ("survival"). The x-axis shows days since the initial outbreak. The central continuous line represents the probability ( $+\mathrm{s}$ indicate recorded outbreaks) and the shaded area represents $95 \%$ confidence intervals ( $\mathrm{n}=34$ herds that had FMD outbreaks and were tracked longitudinally). b. Perceived impact of seven common livestock diseases and syndromes in northern Tanzania measured by pairwise ranking in three livestock management systems ranked by overall importance $(E C F=$ East Coast fever $)(n=35$ agropastoral, 41 pastoral and 23 rural smallholder households). c. Proportion of animals that households reported to show clinical signs of FMD in their livestock by species and age group. Bars represent $95 \%$ confidence intervals ( $n=4852$ animals belonging to 45 households that had FMD outbreaks). d. Effect of FMD outbreaks on cow milk production. Density plots showing cow milk production in three management systems during and outwith FMD outbreaks as reported in household-level interviews. Grey fill $=$ during an FMD outbreak. White fill $=$ without FMD (n=34 agropastoral, 32 pastoral and 20 rural smallholder households). 
Waves of endemic foot-and-mouth disease in eastern Africa suggest feasibility of proactive vaccination approaches

FMD dynamics in livestock FMD control, informed by a comprehensive understanding of the disease epidemiology in eastern Africa, therefore has the potential to reduce vulnerability through increased milk and more efficient crop production. However, understanding FMD epidemiology has suffered from a lack of information on circulating variants. We addressed this knowledge gap through (1) cross-sectional serological surveys across five districts analysed by a Bayesian model to infer the most recent serotype for the period before virus isolation results became available (2011, Fig. 3a and Supplementary Table 5), (2) intensive longitudinal outbreak investigations (2012-2015, Fig. 3b), and (3) collation of eastern African virus typing results from the literature (2008-2015, Fig. 3c and Supplementary Table 6). Model inference from the cross-sectional serology indicated that SAT1, O and SAT2 had passed through the herds before the study (Fig. 3a and Supplementary Table 5). Four serotypes were isolated from cattle outbreaks in Serengeti district during the study period (2012-2015, Fig. 3b): A ( $\mathrm{n}=26$ isolates), $\mathrm{O}(\mathrm{n}=11), \mathrm{SAT} 1(\mathrm{n}=50)$ and SAT2 $(\mathrm{n}=23)$, which were predominantly related to other eastern African viruses from the literature (Supplementary Fig. 1). Collectively, these two analyses showed a sequence of epidemics of serotype SAT1 (2010 to early 2011), O (mid- to late 2011), SAT2 (late 2011 to mid-2012), and A (mid-2012 to mid-2013) before SAT1 returned in late 2013 (Fig. 3a,b) in our study herds. The same pattern repeated itself across eastern Africa more broadly in our meta-analysis of the published literature (Fig. 3c and Supplementary Table 6), with a permutation test of the eastern African data $(\mathrm{n}=265$ typed outbreaks across the region, Supplementary Table 6) showing that outbreaks clustered in time and space in such waves $\left(\mathrm{p}<<10^{-6}\right)$. A more detailed analysis of the longitudinally tracked herds in this study ( $\mathrm{n}=12$ pairs of sequentially typed outbreaks in the same herd, Supplementary Table 7) showed that the sequence of serotypes was not random, with the same serotype never returning immediately $(\mathrm{p}<0.05)$. Although no directionality could be discerned across the sparsely-sampled eastern Africa region as a whole, outbreaks in our most intensively-sampled district spread east-northeast at between 2.6 and $13.1 \mathrm{~km} / \mathrm{month}$ (Fig. 3b, Supplementary Table 8).

Role of wildlife A second, critical gap preventing the development of control measures in eastern Africa is in the understanding of the role of buffalo in FMD livestock epidemiology. This was studied through (1) contemporaneous sympatric cross-sectional sero-surveys of cattle and buffalo in 2011, (2) a comparison of all regional viral sequences from cattle and buffalo in the World Reference Laboratory for FMD's (WRLFMD) repository, (3) household questionnaire data to determine risk factors for cattle sero-positivity, and (4) a case-control study of cattle outbreaks. Serotype dominance in cattle antisera was associated with the serotype involved in the most recent sweep of cattle outbreaks in the district rather than the dominant serotype in the adjacent buffalo population. Relative seroprevalence of the four serotypes in cattle and buffalo antisera differed in four of the five district groups (Fig. 4), with O and SAT1 dominating in cattle and buffalo, respectively. Although SAT1 had highest seroprevalence in cattle in one district, WRLFMD has found no close genetic relationships between any Tanzanian cattle and buffalo isolates for these serotypes (Supplementary Fig. 1, Supplementary Table 9), in contrast to the situation in southern Africa $^{23}$, where FMD has been heavily controlled in cattle through prophylactic vaccination with tailored polyvalent vaccines for many years, leaving buffalo the main remaining reservoir of disease. 
proactive vaccination approaches

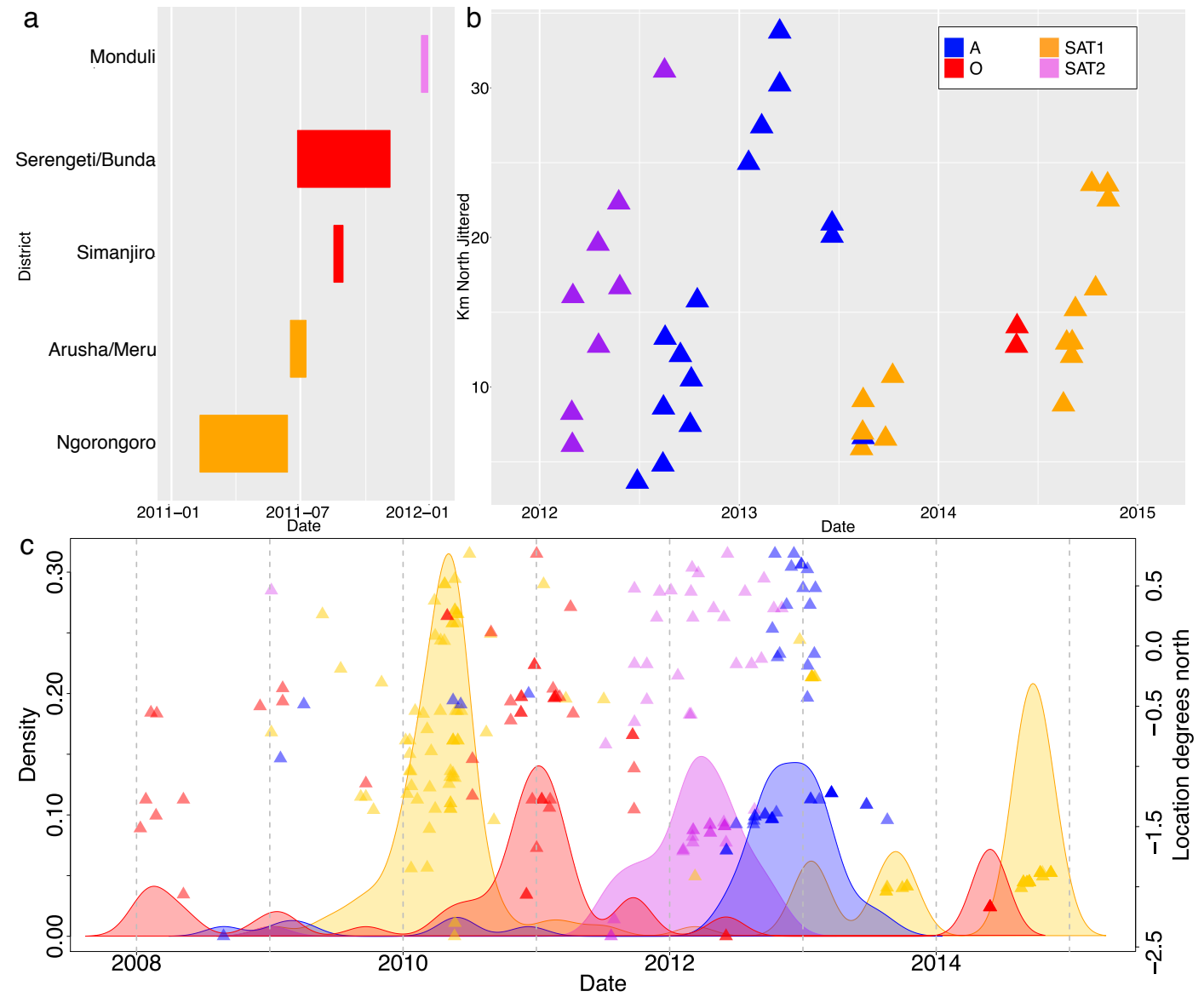

Figure 3: Foot-and-mouth disease virus serotype frequency over time in eastern African cattle. a. Bayesian inference of historical infection from cross-sectional serology in northern Tanzania before virus isolation results were available. The serotype with the highest probability of most recently occurring in each district is plotted against serum sampling period ( $\mathrm{n}=63$ herds). $\mathbf{b}$. Virus isolation, molecular serotyping results and antigen enzyme-linked immunosorbent assay (ELISA) results from Serengeti District (where outbreak investigation efforts were most intensive) between 2012 and 2015 (n=38 FMD outbreaks in 27 herds). c. Density plot (left hand axis) showing results by serotype from virus isolation, molecular serotyping and antigen ELISA for northern Tanzania during 2011-2015 combined with published results from southern Kenya from 2008-2013 (Supplementary Table 8), and a plot showing the same results against latitude (right hand axis). Blue=serotype A, red=serotype $\mathrm{O}$, yellow=serotype SAT1, violet=serotype SAT2 ( $\mathrm{n}=265$ FMD outbreaks).
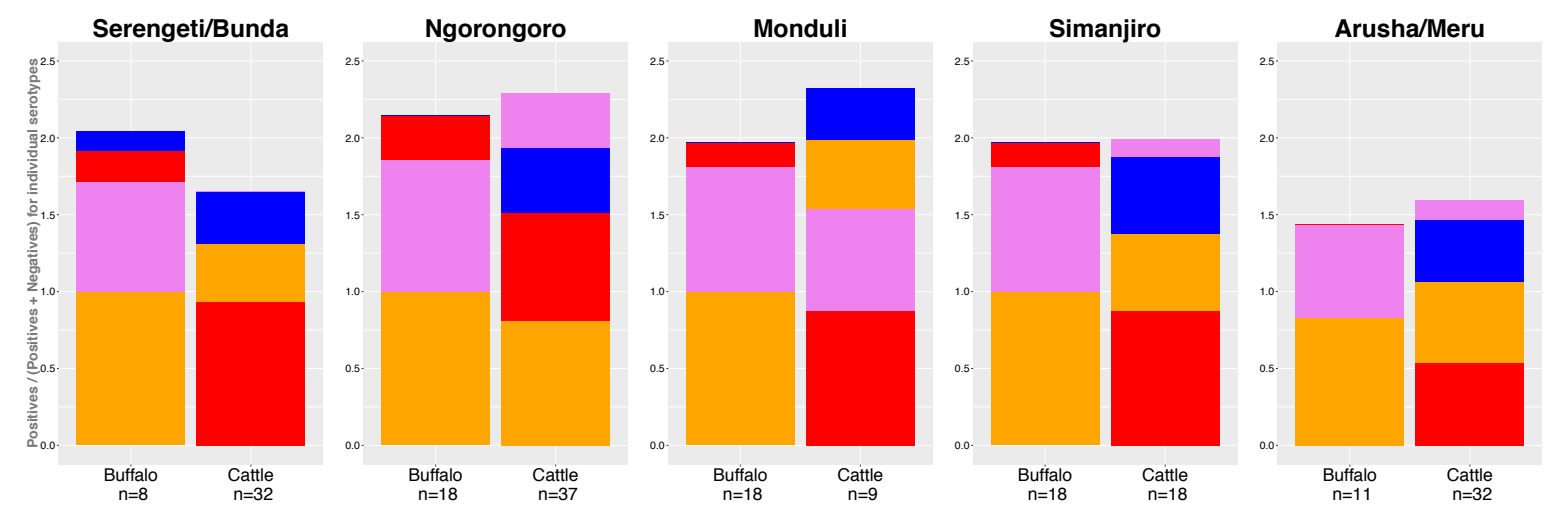

Figure 4: Serum virus neutralisation testing results in buffalo (Syncerus caffer) and cattle. Buffalo (left of each subplot) and cattle (right of each subplot) are grouped according to district group ( $\mathrm{n}=$ total number of samples tested). As Simanjiro and Monduli cattle were sampled adjacent to Tarangire National Park, the same buffalo data were used for comparison in these two areas. Each block of colour represents the seroprevalence for that serotype (proportion positives out of positives plus negatives, excluding inconclusive results). Blue $=$ serotype A, red = serotype O, yellow = serotype SAT1, violet = serotype SAT2. 
Waves of endemic foot-and-mouth disease in eastern Africa suggest feasibility of

proactive vaccination approaches

Although SAT2 was the second most prevalent serotype in buffalo, and spillover in both directions between cattle and buffalo has been reported in southern Africa ${ }^{24}$, the eastern African buffalo SAT2 sequences available in the WRLFMD were not closely related to cattle SAT2 sequences (Supplementary Fig. 1, Supplementary Table 9).

The low seroprevalence of serotypes $\mathrm{O}$ and $\mathrm{A}$ in buffalo (Fig. 4) most likely reflected occasional cattle-to-buffalo spillover or serological cross-reactivity (Supplementary Fig. 2, crossreaction between $\mathrm{O}$ assay and SAT2 serotype is 0.62 ). Outside of experimental infections ${ }^{25}$, serotypes $\mathrm{O}$ and A have never been isolated from buffalo (Supplementary Fig. 1, Supplementary Table 9), suggesting that buffalo are not epidemiologically important for these serotypes. In our risk factor analysis, no wildlife-related predictors were noteworthy in explaining livestock seropositivity, including distance to protected areas and sightings of buffalo and other wildlife (Supplementary Table 10). Conditional logistic regression analysis of outbreak case-control data from agro-pastoral areas revealed that, again, measures of potential contact with buffalo or with other FMD susceptible wildlife did not add to the explanatory power of the model (Supplementary Table 11).

Control through vaccination FMD control was explored through a vaccine matching study by WRLFMD on isolates of all serotypes recovered from the study area. The standard technique for vaccine matching for FMD was followed ${ }^{26}$, providing an " $r$ " serological relationship value between 0 and 1 , with high values indicating a good match between the vaccine and field strain, and low values indicating a significant mismatch and the possibility of vaccine failure. The standard cut-off for a vaccine to be considered well-matched is 0.3 . Existing $\mathrm{O}$ and SAT2 vaccines offered $\mathrm{r}_{1}$ values consistent with protection against all isolates of these serotypes $\left(r_{1} \geq 0.3\right.$; Supplementary Table 12). For A and SAT1, existing vaccines provided $r_{1}$ values that were matching (10/15) or were consistent with protection. No routine polyvalent vaccines on the market offer these strains in combination, but they are available individually as high-potency vaccines, which require a functioning surveillance system to be able to identify the appropriate vaccine to use. While recognising the limitations of $r_{1}$ values as a mechanism for determining protection (and in vivo and field studies should be used to confirm these results), our findings are promising given that these are high-potency vaccines that are likely to protect even with low $\mathrm{r}_{1}$ values ${ }^{27}$. We have also previously shown that tailored vaccines with strains specifically chosen for the region would be expected to perform even better ${ }^{28}$, if resources were available for their development.

\section{Discussion}

In conclusion, our study demonstrates that (1) the production impacts of frequent FMD outbreaks in traditional livestock systems have important negative consequences for the rural poor; (2) a sequence of serotype-specific epidemics has swept through cattle in the eastern Africa region, with a particular serotype dominant and unchanging during an epidemic, in such a way that early typing of outbreak samples would correctly inform the choice of the vaccine serotype; and (3) cattle rather than wildlife appear to drive most FMD transmission. Although focussing on eastern Africa, we believe that our conclusions are probably relevant to other FMD-endemic regions of the continent because we target an area which has the highest buffalo densities in Africa. As a result, if wildlife were involved in FMD epidemiology in an endemic setting, it should be apparent here. Control measures that focus on livestock are therefore likely to be effective, feasible and have less environmental impact than the wildlife-separation approaches used in southern Africa ${ }^{12}$.

An enduring problem is that of resources, making routine prophylactic vaccination infeasible, which has resulted in no high-quality tailored polyvalent vaccine being developed and a concomitant 
lack of faith in those that have been procured. However, our results indicate that targeted serotypespecific livestock vaccination with monovalent high-potency vaccines ahead of oncoming waves of infection could be more affordable and still has the potential to mitigate the economic and disease impacts in the region, contributing to current poverty alleviation agendas ${ }^{29}$.

Indeed, community- and policy-level assessments involving local- and national-level stakeholders have all identified vaccination as the most important prevention mechanism (Supplementary Notes 1 and 2), but in our surveys only 5\% of households vaccinated livestock against FMD. Workshop participants identified major barriers to vaccination in Tanzania as a lack of availability of high-quality polyvalent vaccines tailored to circulating FMD viral strains and the absence of effective policies and strategies for FMD vaccine sourcing, quality control, importation and delivery. However, we show that existing high-potency vaccines should provide protection against each circulating serotype. Finally, policy changes at the inter-governmental level emphasise commodity-based trade ${ }^{30}$ and are moving toward greater recognition of FMD-free compartments based on common risk management rather than on geography ${ }^{31}$. Importantly, this increases market opportunities for livestock products, and the incentive to control FMD and pay for vaccines, contributing to the FAO/OIE's global PCP-FMD strategy.

\section{Methods}

\section{Field studies}

Field studies were conducted in livestock-wildlife interface areas of northern Tanzania during 20112014, including nine districts spread across three ecosystems (Fig. 1): Serengeti (including Serengeti, Bunda, Ngorongoro and Longido districts), Tarangire (including Simanjiro and Monduli districts) and Arusha (including Arusha, Arusha Urban and Meru districts). The study sites were representative of three livestock management practices: agropastoral, pastoral, and rural smallholder. Research approval for the study was granted by the Tanzania Commission for Science and Technology (COSTECH) - permits numbers 2010-385-ER-90-15, 2012-182-ER-90-15, 2013, study 338-ER2010-129 and 2015-92-ER-2015-81. Informed written consent was obtained from all participants before commencing household questionnaires or animal sampling. Survey design, animal sampling and sample management are described in detail in the Supplementary Methods.

The project comprised six inter-related field studies (A - F) (Supplementary Table 13).

Study A: Livestock cross-sectional study. Data were collected from a stratified random sample of 85 livestock owning households in 40 villages in the proximity of wildlife protected areas. Livestock from each household were clinically examined and serum sampled $(n=1410$ cattle, 877 goats and 451 sheep) to obtain FMD seroprevalence data. Questionnaires were conducted to collect data about socioeconomic impacts of FMD at household level, and potential risk factors for FMD seropositivity in livestock (Supplementary Table 13, study A). One household, which had livestock serum sampled but did not complete the questionnaire, was excluded from analyses.

Study B: Buffalo cross-sectional study. Serum and oropharyngeal fluid samples (OPFs) were collected from buffalo in adjacent wildlife areas: Arusha ( $\mathrm{n}=23$ sera, $25 \mathrm{OPFs})$, Serengeti $(\mathrm{n}=36$ and 36), and Tarangire ( $\mathrm{n}=24$ and 25) National Parks, and the Ngorongoro Conservation Area $(\mathrm{n}=116$, 85) (Fig. 1, Supplementary Table 13, study B).

Study C: Outbreak investigations. An outbreak tracking and investigation study based on active surveillance was implemented in one of the study districts, Serengeti (Fig. 1), to obtain clinical 
Waves of endemic foot-and-mouth disease in eastern Africa suggest feasibility of

proactive vaccination approaches

material from FMD outbreaks for diagnosis and serotype/variant characterisation. Survey data collected from the affected households and herds were examined to determine the morbidity and mortality associated with the outbreaks. Detailed follow-up questionnaires were conducted in 17 of the 50 households affected by FMD outbreaks to better understand outbreak impacts (Supplementary Table 13, study C).

Study D: Longitudinal monitoring of outbreak herds. Of the outbreak herds, 34 were tracked longitudinally over more than one visit. Serial outbreaks were recorded in 15 of these herds (Supplementary Table 13, study D).

Study E: Case-control study. A case-control study, stratified at village level ( $\mathrm{n}=70$ households in 7 villages), was implemented in Serengeti District to investigate herd-level risk factors for FMD outbreaks in cattle. Through the outbreak investigation platform (study C), villages undergoing FMD outbreaks were identified. Case households were defined as those whose cattle herds displayed lesions characteristic of FMD during the outbreak. In a subset of households, cattle reported with FMD were investigated through lesion sampling and laboratory diagnostic testing with all households confirmed positive. In control households, selected in the same village, no lesions were observed in cattle during the outbreak, and for six weeks after the initial outbreak investigation visit. The risk period was one month before the first outbreak in the village. Five case and five control households were selected randomly from the list of all affected and unaffected herds in each of the seven villages during the risk period. Questionnaire surveys were conducted to obtain information about potential risk factors for outbreaks during the risk period including herd size, livestock movements and contacts with other livestock, people or wildlife (Supplementary Table 13, study E).

Study F: Prospective longitudinal study. A prospective longitudinal study involved herds of cattle monitored through serial FMD outbreaks with the objective of characterising the serological response to FMD infections. Two herds of 100 cattle were tracked longitudinally ${ }^{32}$, including daily inspection, regular clinical examinations and serum sampling. One of these herds was tracked through four FMD outbreaks between January 2011 and November 2013, with 19 serum sampling time points. The second herd was tracked between December 2011 and March 2013, suffered only one FMD outbreak, and was serum sampled at eight time points (Supplementary Table 13, study F).

\section{Laboratory methods}

Laboratory testing was performed at WRLFMD and is summarised in Supplementary Table 13 for each component of the study.

Serum antibodies against FMDV non-structural proteins (NSPs), which are indicative of viral replication for all serotypes, were detected with a commercial blocking ELISA (PrioCHECK FMDV NS). Results of percentage inhibition (PI) $\geq 50 \%$ were considered positive. Serotype-specific serum antibodies were detected using a virus neutralisation test $(\mathrm{VNT})^{26}$ using virus isolates (serotypes $\mathrm{O}$, A, SAT1 and SAT2) from the study area. Titres $>32$ were considered positive, between 16 and 32 inconclusive and $<16$ negative $^{26}$. The Supplementary Methods describe the method used to select sera for VNT (Supplementary Tables 13, studies A, B and F).

Serotype-specific serum antibodies were also measured using an in-house solid phase competition ELISA (SPCE) based on the structural proteins (SPs) from serotype O, SAT1 and SAT2 FMDV isolates from the study area. For the serotype O SPCE, a PI of $\geq 50 \%$ represented a positive result, whereas for the SAT SPCEs a PI $\geq 40 \%$ was considered positive. A commercial blocking ELISA was used to detect antibodies against the structural proteins of FMDV serotype A (PrioCHECK ${ }^{\text {TM }}$ FMDV 
Waves of endemic foot-and-mouth disease in eastern Africa suggest feasibility of proactive vaccination approaches

Type A Antibody ELISA Kit, ThermoFisher Scientific), with a PI $\geq 50 \%$ considered positive (Supplementary Tables 13, studies A and F).

FMD lesion samples ( $\mathrm{n}=159$ from 62 outbreak investigations) were analysed by virus isolation, antigen typing and sequencing of the VP1 viral protein (Supplementary Tables 13, studies C, D and F).

Vaccine matching was carried out for 20 viruses ( $\mathrm{n}=8$ serotype A, 2 O, 7 SAT1 and 3 SAT2) isolated during this study according to the protocol outlined within the OIE Manual ${ }^{26}$. A relationship coefficient, $\mathrm{r}_{1}$-value, was calculated by dividing the heterologous neutralisation titre (field strain against the vaccinal serum) by the homologous neutralisation titre (vaccine strain against the vaccinal serum) using a two dimensional VNT ${ }^{26}$. Five virus doses (ranging from 10 to 1000 Tissue Culture Infectious Dose $50\left[\mathrm{TCID}_{50}\right]$ ) were tested against a serial two-fold dilution of serum. From each of these doses the neutralisation titre was calculated and a regression line was drawn. From the regression, the neutralisation titre required for $50 \%$ neutralisation of $100 \mathrm{TCID}_{50}$ virus dose was calculated. Vaccine matching results are shown in Supplementary Table 12.

\section{Analyses}

Descriptive statistics. Income sources were described using data from the cross-sectional (Supplementary Table 13, study A) and outbreak studies (Supplementary Table 13, study C). Seropositivity levels were estimated from the cross-sectional study (Supplementary Table 13, study A), and morbidity and mortality from outbreak investigations (Supplementary Table 13, study C). Where proportions were reported, $95 \%$ confidence intervals were generated using the exact binomial method.

Phylogenetic analysis. The evolutionary history was inferred using the Maximum Likelihood method based on the General Time Reversible (GTR) (SAT 1 and SAT 2) or Tamura-Nei (TN93) (O and A) models as implemented in MEGA 6.06 ${ }^{33}$. Branching confidence was measured using 1000 bootstrap pseudo-replicates. The trees with the highest log likelihood are shown (Supplementary Fig. 1). A discrete gamma distribution was used to model evolutionary rate differences among sites [five categories $(+\mathrm{G})]$. The rate variation model allowed for some sites to be evolutionarily invariable $[(+\mathrm{I})]$.

Frequency of FMD outbreaks. Data from 34 herds tracked longitudinally were used for survival analysis. A Kaplan Meyer survival curve (Fig. 2a) was generated and $25^{\text {th }}, 50^{\text {th }}$ and $75^{\text {th }}$ quantiles for time between outbreaks were estimated.

Household perception of the relative importance of different livestock diseases. Households were asked to identify and rank seven livestock diseases/syndromes known to be present in the area in order of importance (Fig. 2b).

A pairwise ranking algorithm was developed to compare the perceived importance of each disease. Knowledge of and ranking of each of the seven diseases and disease syndromes $\left(\tau_{k}\right)$ by livestock owners was compared with that for the other six $\left(\tau_{j}\right)$. Pairwise ranking scores $\left(P_{k j}\right)$ were produced for every possible pairwise combination of diseases for every household. 
Waves of endemic foot-and-mouth disease in eastern Africa suggest feasibility of proactive vaccination approaches
$P_{k j}=\left\{\begin{array}{c}\tau_{k} \text { known } \\ \tau_{k} \text { unknown, } \tau_{j} \text { known } \\ \text { neither known }\end{array}\right.$
(1 if $\tau_{j}$ unknown or $\tau_{j}$ ranked below $\tau_{k}$
$\mathrm{NA}=$ Not Answered
0.5 if $\tau_{j}$ and $\tau_{k}$ ranked equally
0 if $\tau_{j}$ ranked above $\tau_{k}$
0
NA

For agropastoral, pastoral and smallholder livestock practices, pairwise ranking scores for each disease against all the other diseases, $\tau_{k} P \tau_{j}$, were summed and divided by the number of pairwise comparisons $\left(n_{k}\right)$ between that disease and the others to produce an average pairwise score per comparison $\left(v_{k}\right)$.

$$
v_{k}=\sum_{\substack{j: P_{k j} \neq N A \\ 0 \leq v_{k} \leq 1}} \frac{P_{k j}}{n_{k}}
$$

$n_{k}=$ Number of non-NA pairwise comparisons between $\tau_{k}$ and any other disease

For plotting purposes (Fig. $2 \mathrm{~b}$ ), the neutral pairwise comparison score of 0.5 was subtracted from $v_{k}$ for each disease to highlight whether the disease was ranked higher (positive value) or lower (negative value) than this.

Economic impacts of FMD. Economic analyses followed a microeconometric approach to the agricultural household model, including household production relationships and household expenditure $^{34}$. Separate regression models were applied to quantify the impact of FMD events on milk production (ordinary least squares), number of lactating cows (Poisson regression), traction (logistic regression), and livestock sales (Tobit regression), as well as education expenditures (Tobit regression) and human health (Heckman regression). Specification of each regression model was dependent on the distribution of the dependent variable, censoring of the dependent variable, presence of zero observations, and the nature of the survey data. Marginal effects were calculated to represent economic responsiveness and interpret economic outcomes. Robust standard errors were calculated to account for heterogeneity across the households. Additional details are presented in Supplementary Tables 3 and 14-17.

Risk factor analyses. A generalised linear mixed-effects model (GLMM) with a logit link function was used to investigate the effects of explanatory variables on the likelihood of a positive NSP ELISA result. After initial descriptive analyses, seven potential explanatory variables were selected for the initial trial model based on the strongest biological rationale: 1) animal age, 2) species, 3) livestock practice, 4) herd size, 5) maximum time walked to reach grazing and water, 6) wildlife sightings (with separate categories for buffalo, non-buffalo FMD susceptible wildlife and non-susceptible wildlife), 7) proximity to a wildlife protected area containing buffalo, and 8) acquisition of livestock in the past four months.

For model selection, variables were dropped in a stepwise fashion with the least significant variable upon likelihood ratio testing (LRT) being dropped first. For each step, the LRT was repeated for the remaining variables.

Power analysis for the cross-sectional study was performed retrospectively by simulation ${ }^{35}$. Simulations of between 1000 and 5120 livestock sampled from between 40 and 160 herds were made 
Waves of endemic foot-and-mouth disease in eastern Africa suggest feasibility of

proactive vaccination approaches

and buffalo sighting data were randomly generated based on a Bernoulli distribution and with a probability of 0.5 of a buffalo sighting weekly or more often. Simulated village levels were generated on the basis of two herds per village. A scenario was investigated where the baseline probability of livestock being positive for NSP antibodies was 0.5 based on FMDV sero-prevalence estimates from Tanzania, Uganda and Kenya. Simulated effects of buffalo sightings were created where weekly buffalo sightings by the household increased the probability of their livestock being seropositive by between 0 and 0.45 (increased the odds by a ratio between 1 and 19). A variance of 1 was assumed for the herd and village level random effects. For 2688 livestock from 84 herds and 42 villages, when buffalo sightings had no effect, Wald $\mathrm{p}$ values were less than 0.05 for $6 \%$ of simulations. When buffalo sightings increased the probability of livestock in the herd being seropositive by 0.2 , Wald $\mathrm{p}$ values were less than 0.05 for $85 \%$ of simulations. When the probability increased by 0.25 , $\mathrm{p}$ values were less than 0.05 for $96 \%$ of simulations.

Potential explanatory variables for FMD outbreaks in the case-control study were investigated using a conditional logistic regression model with village level strata. The following variables from within the risk period of month prior to the first outbreak in the village were incorporated: 1) herd size, 2) newly acquired animals, 3) sightings of buffalo and other wildlife near the livestock herd, 4) grazing or watering in different areas from usual, 5) a measure of livestock contacts during grazing and watering (Supplementary Methods), 6) a measure of livestock contacts during dipping (Supplementary Methods) and 7) a measure of visitors to the herd (Supplementary Methods).

Similarly to the GLMM, model selection for the conditional logistic regression model was based on likelihood ratio testing, with the variables adding least to the explanatory ability of the model being dropped first. Analysis of the statistical power of the model was performed retrospectively. A simulated dataset with an exposure level of $50 \%$ for buffalo sightings was generated. An odds ratio of 3 for being a case in association with weekly buffalo sightings was simulated. This simulation was repeated 10,000 times to estimate the power of the case control study. For a study with 35 cases and 35 controls, the power estimated from this calculation was $59 \%$.

Characterisation of the serological response to infection in an endemic multi-serotype FMD environment. Inferring from serology the most recent FMDV serotype infecting an animal can be challenging in a multi-serotype environment, because the animal may have residual seropositivity from previous infections or an anamnestic immune response to other previously encountered serotypes. In addition, cross-reactivity between serotypes in FMD antibody-based assays is well recognised ${ }^{36-39}$. To address this issue, a Bayesian model of NSP and SP ELISA reactivity dynamics (Supplementary Table 18) was trained using data on the timing of individual outbreaks and associated clinical lesions (Supplementary Tables 13, studies C and D), and from serological and virus typing testing generated from an intensively sampled herd tracked longitudinally ( $\mathrm{n}=100$ cattle, Supplementary Table 13, study F) that suffered four serial outbreaks over three years. Virus isolation and typing data were available from three of these outbreaks, and ELISA results were generated from serum samples collected from the herd at 19 different sampling points over the three years.

The model was conceptually simple, comprising an exponential decay term for NSP (Supplementary Fig. 3) and SP (Supplementary Fig. 4) ELISA reactivities for each animal between infections, with two half-lives, one for NSP and one collectively for all of the SP ELISAs ( $\omega$ and $\widehat{\omega}$, respectively, Supplementary Table 18b). At the point of infection, there is an instantaneous (relative to the multiyear duration of the model) change in NSP PI to a level $r$, and of SP PI to a level $u$ for the homologous assay, and an increase by a proportion $\gamma_{n, s}$ (the cross-reactivity between the assay, n, and the serotype, 
Waves of endemic foot-and-mouth disease in eastern Africa suggest feasibility of proactive vaccination approaches

$\mathrm{s})$ of the difference between the current PI and $\mathrm{u}$ for heterologous assays. The equations governing these dynamics are found in Supplementary Table 18c. There is then a normally-distributed measurement error imposed on these "true" reactivities $\left(g_{i, j}\right.$ and $\hat{g}_{n, i, j}$, Supplementary Table 18b). The timing and serotype of outbreaks inferred for the cross-sectional herds, as well as the infection status of every individual animal in each outbreak, are represented by $\chi_{h}, \varphi_{h}$, and $f_{i, k}$, respectively (Supplementary Table 18b), with the priors of the other parameters and hyper-parameters of the model also found in the same table. The hierarchical, auto-regressive, mixture model therefore takes herd-level outbreak events, animal-level infection, ELISA reactivity decay and cross-reactivity in the ELISA assays into account.

A minimum of four Markov Chain Monte Carlo (MCMC) chains was used for each model. As well as visual assessment of the MCMC traces for each parameter in the model, convergence of the different chains was summarised with the potential scale reduction factor (PSRF, ratio of betweenchain variance to within-chain variance). A PSRF value of 1.1 or less in combination with visual observation of convergence was considered to represent acceptable convergence between chains for each parameter. Models were selected on the basis of convergence with the training data and on initial validations performed by removing information about outbreak time, serotype and which animals were infected from the data fed into each model and testing their ability to infer these from longitudinal serology data alone. As a second validation, a serological dataset from a different herd that had not been used for model training was used. This came from eight sampling points over a 14month period of a second herd of 100 animals that suffered one FMD outbreak. The longitudinal serological data alone were fed to the model, and its ability to infer outbreak time, serotype and infected animals was again tested. Finally, the model was validated with cross-sectional serological data from single time points, where the infection history of the animal was known. The validation results are presented in Supplementary Tables 19 and 20. The model was then applied to crosssectional serological data to infer FMD infection history at district level (Fig. 3a and Supplementary Table 5) and cross-reactivity between serotypes (Supplementary Fig. 2).

Analyses to understand patterns of FMD over space and time. To understand serotype-specific patterns of FMD infection over space and time, we focused on the area (Serengeti District) for which the highest number of virus typing results ( 85 results from 38 outbreaks) over the longest time window (February 2012 to November 2014) were available. Data from 14, 2, 15 and 8 serotype A, O, SAT1 and SAT2 outbreaks were available, respectively. The association between outbreak location and date was investigated for serotypes A, SAT2 and the second of the SAT1 waves ( 9 outbreaks). Kilometres northwards and kilometres eastwards for outbreaks over time were positively correlated with each other (Pearson's $r=0.82$ for A, 0.97 for SAT1 and 0.76 for SAT2), with the timing of the outbreaks suggesting that new cases arose in a broadly east-northeasterly direction over time. Therefore, principal component analysis was used to find the best fitting direction of travel of each of the different serotype wave fronts (Supplementary Table 8).

We subsequently investigated the consistency of FMDV serotype circulation patterns and serotypic dominance over a broader geographic scale. The PubMed database (http://www.ncbi.nlm.nih.gov/pubmed/) was reviewed using the search terms "foot-and-mouth disease", "cattle" and each of "Kenya", "Tanzania", and "Uganda". Articles from this search that reported virus isolation or virus typing results after 2008 were identified and summarised. Where sample collection dates and locations were available in association with virus typing results, these were collated for comparison to the results from the present study. The WRLFMD database ${ }^{40}$ was 
Waves of endemic foot-and-mouth disease in eastern Africa suggest feasibility of proactive vaccination approaches

also searched for results from Kenya, Tanzania and Uganda. The WRLFMD records from 2010 onwards had location data readily available; therefore these were also included. Supplementary Table 9 shows the sources used for virus typing data. The highest density typing data in time and space came from southern Kenya and northern Tanzania between 2008 and 2015. These data were therefore brought forward for analyses.

Randomisation test. Fourteen of the herds from Serengeti District and a further herd from Simanjiro were tracked through multiple FMD outbreaks. Of these, eight had a total of twelve pairs of sequential outbreaks where both outbreaks were successfully serotyped (with a total of four serotypes, Supplementary Table 7). None of the eight herds suffered sequential outbreaks of the same serotype, which would fail to occur by chance with probability $\left(\frac{3}{4}\right)^{12}<0.05$.

A total of 265 FMD virus typing results were available from northern Tanzania and southern Kenya between 2008 and 2014 (Fig. 3c). Waves of different serotypes swept through the region over time: between 2010 and 2014 SAT1, O, SAT2 and A were the dominant serotypes to cause outbreaks in sequential order. To test whether the sequence of serotypes over time was random, a randomisation test was utilised. The sequence of serotypes causing the 265 outbreaks was randomly resampled, and the number of outbreaks of the same serotype following each other was counted. The true dataset had 206 consecutive outbreaks of the same serotype out of 264 pairs of outbreaks. The highest number of consecutive outbreaks from two million iterations of the randomisation was 116, supporting the hypothesis that the observed waves of serotype dominance were not random.

\section{Code Availability}

The analyses used for the current study are described in the Analyses subsection of the Methods, and code is available from the corresponding author on reasonable request.

\section{Data Availability}

The datasets generated during and/or analysed during the current study and the associated code are either available from GenBank (sequences, see Supplementary Table 9) or from the corresponding author on reasonable request.

\section{References}

1. Tekleghiorghis, T., Moormann, R. J. M., Weerdmeester, K. \& Dekker, A. Foot-and-mouth disease transmission in Africa: implications for control, a review. Transbound. Emerg. Dis. 63, 136-151 (2016).

2. Weaver, G. V, Domenech, J., Thiermann, A. R. \& Karesh, W. B. Foot-and-mouth disease: a look from the wild side. J. Wildl. Dis. 49, 759-785 (2013).

3. Food and Agriculture Organization of the United Nations (FAO)/Office International des Epizooties - World Organisation for Animal Health (OIE)/European Comission for the control of Foot-and-Mouth Disease (EuFMD). The progressive control pathway for foot-andmouth disease control (PCP-FMD). (2012). Available at:

http://www.oie.int/eng/A_FMD2012/docs/Background_papers_PCP_FMD.pdf (Accessed: 5th July 2017)

4. Sumption, K., Domenech, J. \& Ferrari, G. Progressive control of FMD on a global scale. Vet. Rec. 170, 637-9 (2012).

5. Perry, B. \& Grace, D. The impacts of livestock diseases and their control on growth and 
Waves of endemic foot-and-mouth disease in eastern Africa suggest feasibility of proactive vaccination approaches

development processes that are pro-poor. Philos. Trans. R. Soc. Lond. B. Biol. Sci. 364, 2643-55 (2009).

6. Otte, J. et al. Livestock sector development for poverty reduction: an economic and policy perspective: livestock's many virtues. Food and Agriculture Organization of the United Nations (2012). Available at: http://www.fao.org/docrep/015/i2744e/i2744e00.pdf (Accessed: 5th July 2017)

7. Perry, B. D. \& Rich, K. M. Poverty impacts of foot-and-mouth disease and the poverty reduction implications of its control. Vet. Rec. 160, 238-241 (2007).

8. Knight-Jones, T. J. D. \& Rushton, J. The economic impacts of foot and mouth disease - What are they, how big are they and where do they occur? Prev. Vet. Med. 112, 161-173 (2013).

9. World Bank. World Development Indicators database. (2017). Available at: http://databank.worldbank.org/data/download/GDP.pdf (Accessed: 20th August 2017)

10. Pendell, D. L., Marsh, T. L., Coble, K. H., Lusk, J. L. \& Szmania, S. C. Economic assessment of FMDv releases from the National Bio and Agro Defense Facility. PLoS One 10, e0129134 (2015).

11. Miguel, E. et al. Contacts and foot and mouth disease transmission from wild to domestic bovines in Africa. Ecosphere 4, 51 (2013).

12. Brückner, G. K. et al. Foot and mouth disease: the experience of South Africa. Rev. Sci. Tech. 21, 751-64 (2002).

13. Naranjo, J. \& Cosivi, O. Elimination of foot-and-mouth disease in South America: lessons and challenges. Philos. Trans. R. Soc. Lond. B. Biol. Sci. 368, 20120381 (2013).

14. Paton, D. J., Sumption, K. J. \& Charleston, B. Options for control of foot-and-mouth disease: knowledge, capability and policy. Philos. Trans. R. Soc. B Biol. Sci. 364, 2657-2667 (2009).

15. East, R. \& Estes, R. D. African Antelope Database 1998. (International Union for the Conservation of Nature Species Survival Commission; Gland : IUCN, 1999).

16. Paton, D. J. \& Taylor, G. Developing vaccines against foot-and-mouth disease and some other exotic viral diseases of livestock. Philos. Trans. R. Soc. Lond. B. Biol. Sci. 366, $2774-$ 81 (2011).

17. Agriculture and Consumer Protection Department, Animal Production and Health, Food and Agriculture Organization of the United Nations. Global Livestock Densities. (2011).

Available at: http://www.fao.org/ag/againfo/resources/en/glw/GLW_dens.html (Accessed: 10th May 2011)

18. Robinson, T. P. et al. Mapping the global distribution of livestock. PLoS One 9, e96084 (2014).

19. Bedelian, C., Nkedianye, D. \& Herrero, M. Maasai perception of the impact and incidence of malignant catarrhal fever (MCF) in southern Kenya. Prev. Vet. Med. 78, 296-316 (2007).

20. Jost, C. C. et al. Epidemiological assessment of the Rift Valley fever outbreak in Kenya and Tanzania in 2006 and 2007. Am. J. Trop. Med. Hyg. 83, 65-72 (2010).

21. Lawson, D. W. et al. Ethnicity and child health in northern Tanzania: Maasai pastoralists are disadvantaged compared to neighbouring ethnic groups. PLoS One 9, e110447 (2014).

22. Knight-Jones, T. J. D., McLaws, M. \& Rushton, J. Foot-and-Mouth Disease Impact on Smallholders - What Do We Know, What Don't We Know and How Can We Find Out More? Transbound. Emerg. Dis. 64, 1079-1094 (2017)

23. Vosloo, W., Boshoff, K., Dwarka, R. \& Bastos, A. D. The possible role that buffalo played in the recent outbreaks of foot-and-mouth disease in South Africa. Ann. N. Y. Acad. Sci. 969, 187-190 (2002).

24. Brito, B. P. et al. Transmission of foot-and-mouth disease SAT2 viruses at the wildlifelivestock interface of two major transfrontier conservation areas in southern Africa. Front. 
Microbiol. 7, 528 (2016).

25. Anderson, E. C., Doughty, W. J., Anderson, J. \& Paling, R. The pathogenesis of foot-andmouth disease in the African buffalo (Syncerus caffer) and the role of this species in the epidemiology of the disease in Kenya. J. Comp. Pathol. 89, 541-549 (1979).

26. Office International des Epizooties - World Organisation for Animal Health (OIE). Manual of diagnostic tests and vaccines for terrestrial animals 2016: Chapter 2.1.8: Foot-and-mouth disease (infection with foot-and-mouth disease virus). (2017). Available at: http://www.oie.int/fileadmin/Home/eng/Health_standards/tahm/2.01.08_FMD.pdf (Accessed: 9th July 2017)

27. Brehm, K. E., Kumar, N., Thulke, H. \& Haas, B. High potency vaccines induce protection against heterologous challenge with foot-and-mouth disease virus. Vaccine 26, 1681-1687 (2008).

28. Bari, F. D. et al. Genetic and antigenic characterisation of serotype A FMD viruses from East Africa to select new vaccine strains. Vaccine 32, 5794-5800 (2014).

29. United Nations. Sustainable development goals. (2015). Available at: http://www.un.org/sustainabledevelopment/sustainable-development-goals/ (Accessed: 18th March 2017)

30. Rich, K. M. \& Perry, B. D. Whither commodity-based trade? Dev. Policy Rev. 29, 331-357 (2011).

31. Office International des Epizooties - World Organisation for Animal Health (OIE). Terrestrial Animal Health Code. (2015). Available at: http://www.oie.int/internationalstandard-setting/terrestrial-code/access-online/ (Accessed: 9th July 2017)

32. Lankester, F. et al. A field vaccine trial in Tanzania demonstrates partial protection against malignant catarrhal fever in cattle. Vaccine 34, 831-838 (2016).

33. Nei, M. \& Kumar, S. Molecular evolution and phylogenetics. (Oxford University Press, Oxford, 2000).

34. Marsh, T. L., Yoder, J., Deboch, T., Mcelwain, T. F. \& Palmer, G. H. Livestock vaccinations translate into increased human capital and school attendance by girls. Sci. Adv. 2, e1601410 (2016).

35. Johnson, P. C. D., Barry, S. J. E., Ferguson, H. M. \& Müller, P. Power analysis for generalized linear mixed models in ecology and evolution. Methods Ecol. Evol. 6, 133-142 (2015).

36. Namatovu, A. et al. Challenges for serology-based characterization of foot-and-mouth disease outbreaks in endemic areas; identification of two separate lineages of serotype $\mathrm{O}$ FMDV in Uganda in 2011. Transbound. Emerg. Dis. 62 522-534 (2015).

37. Di Nardo, A. et al. Serological profile of foot-and-mouth disease in wildlife populations of West and Central Africa with special reference to Syncerus caffer subspecies. Vet. Res. 46, 77 (2015).

38. Wekesa, S. N. et al. Characterisation of recent foot-and-mouth disease viruses from African buffalo (Syncerus caffer) and cattle in Kenya is consistent with independent virus populations. BMC Vet. Res. 11, (2015).

39. Hedger, R. S., Barnett, I. T., Gradwell, D. V \& Travassos Dias, P. Serological tests for footand-mouth disease in bovine serum samples. Problems of interpretation. Rev. Sci. Tech. 1, 387-393 (1982).

40. World Reference Laboratory for Foot-and-Mouth Disease (WRLFMD). Molecular epidemiology reports. (2015). Available at: http://www.wrlfmd.org/fmd_genotyping/ (Accessed: 22nd August 2015) 


\section{Acknowledgments}

We are grateful to the Tanzania Commission for Science and Technology, Tanzania Ministry of Livestock and Fisheries, Tanzania Wildlife Research Institute, Tanzania National Parks, and the Ngorongoro Conservation Area Authority for permissions and productive collaborations; the District Veterinary Officers, Livestock Field Officers and Community Animal Health Workers in Mara and Arusha Regions for assistance with data and sample collection, and stakeholder engagement activities; the Frankfurt Zoological Society and Tanzania Conservation Resource Centre for logistical and administrative support in Tanzania; and colleagues in the WRLFMD for their contribution to laboratory analyses. We are indebted to Raphael Mahemba Shabani for his dedication and hard work throughout the study, and Enos Kamani for coordinating field activities in the initial stages of the project. We are grateful to Jonathan Yoder for valuable contributions to the socio-economic surveys and comments on an earlier version of the manuscript. We thank Grant Hopcraft and Mike Shand for assistance with production of maps for this manuscript. This work was supported by the Biotechnology and Biological Sciences Research Council (BBSRC), the Department for International Development and the Scottish Government through the Combating Infectious Diseases of Livestock for International Development initiative (projects BB/H009302/1 and BB/H009175/1). The work of the WRLFMD was supported by the Department for Environment, Food and Rural Affairs (Project SE1143: Defra, UK), and funding provided to the EuFMD from the European Union. Doctoral training for M.C. was funded by a BBSRC Doctoral Training Grant. T.L. and R.R. received support from the Scottish Universities Life Sciences Alliance (SULSA). R.R. was supported by BBSRC grant BB/L004828/1. R.F. and G.N. were supported by the Wellcome Trust-funded Afrique One consortium. Community- and policy-related knowledge exchange initiatives were funded through the Afrique One consortium, contributions by Merck Animal Health to the University of Glasgow, and the Boyd Orr Centre for Population and Ecosystem Health. The African Development Bank funded the Southern African Development Community Transboundary Animal Diseases (SADC TADs) Project at the SADC Secretariat. Opinions, findings, conclusions and recommendations are those of the authors and do not necessarily reflect the views of the funding bodies.

\section{Author contributions}

The study was designed by M.C., R.R., H.A., D.J.P., S.P., D.T.H., T.L.M., S.C. and T.L., field work was carried out by H.A., R.F., R.K., T.K., F.L., A.L., D.M., G.N. and T.L., laboratory work was performed by M.C., N.J.K., K.B.B., V.L.F., D.P.K., A.B.L., F.F.M., K.P., J.W., S.P., S.C. and T.L., modelling and data analysis was conducted by M.C., R.R., U.B., N.J.K. and T.L.M., and the paper was written and revised by all authors.

\section{Competing interests}

A.K. works for Merck Animal Health (known as MSD Animal Health outside USA and Canada), which manufactures FMD vaccines. The workshops described in Supplementary Notes 1 and 2 were funded jointly by the Wellcome Trust through the Afrique One Consortium, the University of Glasgow and its Boyd Orr Centre for Population and Ecosystem Health, and MSD Animal Health. MSD had no control over the design, implementation or analysis of the results of the workshops, and the MSD funding has not influenced the work presented in this manuscript in any way. A.K. had no influence on the study design or the analysis described in the manuscript as a whole, but was consulted in discussions about the feasibility of the proposed solutions and during the manuscript preparation process. 